\title{
Towards Energy-Proportional Computing for Enterprise-Class Server Workloads
}

\author{
Balaji Subramaniam \\ Dept. of Computer Science \\ Virginia Tech \\ balaji@cs.vt.edu
}

\author{
Wu-chun Feng \\ Dept. of Computer Science \\ Virginia Tech \\ feng@cs.vt.edu
}

\begin{abstract}
Massive data centers housing thousands of computing nodes have become commonplace in enterprise computing, and the power consumption of such data centers is growing at an unprecedented rate. Adding to the problem is the inability of the servers to exhibit energy proportionality, i.e., provide energy-efficient execution under all levels of utilization, which diminishes the overall energy efficiency of the data center. It is imperative that we realize effective strategies to control the power consumption of the server and improve the energy efficiency of data centers. With the advent of Intel Sandy Bridge processors, we have the ability to specify a limit on power consumption during runtime, which creates opportunities to design new power-management techniques for enterprise workloads and make the systems that they run on more energy-proportional.

In this paper, we investigate whether it is possible to achieve energy proportionality for an enterprise-class server workload, namely SPECpower_ssj2008 benchmark, by using Intel's Running Average Power Limit (RAPL) interfaces. First, we analyze the power consumption and characterize the instantaneous power profile of the SPECpower benchmark at a subsystem-level using the on-chip energy meters exposed via the RAPL interfaces. We then analyze the impact of RAPL power limiting on the performance, per-transaction response time, power consumption, and energy efficiency of the benchmark under different load levels. Our observations and results shed light on the efficacy of the RAPL interfaces and provide guidance for designing power-management techniques for enterprise-class workloads.
\end{abstract}

\section{INTRODUCTION}

Massive data centers housing thousands of computing nodes have become common. A large fraction of such data centers' total cost of ownership (TCO) comes from the cost of building and maintaining infrastructure that is capable of powering such large-scale data centers and the recurring energy costs 12. Consequently, power and energy have emerged as first-order design constraints in data centers. These issues are further magnified by the inability of servers to provide energy-efficient execution at all levels of utilization. The recent recommendation of energy proportionality in servers, i.e., to design servers which consume power proportional to the utilization, is a move in the right direction as it has the potential to double the energy efficiency of servers 9 . However, achieving energy-proportional operation is a challenging task particularly given that typical servers consume $35-45 \%$ of peak power even when idling.

Typically, dynamic voltage and frequency scaling (DVFS) has been used to achieve better energy efficiency as it can potentially give up to cubic energy savings [17, 24, 26]. However, as we will show in this paper, the subsystem affected by DVFS (i.e., the core ${ }^{1}$ is already the most energyproportional part of the system. There are other subsystems, such as the uncore ${ }^{2}$ that consume constant power, irrespective of the system utilization. In order to achieve energy proportionality, we need to understand the power consumption of each subsystem at different levels of utilization and to leverage mechanisms that enable us to control the power consumption of these subsystems.

With the advent of Intel Sandy Bridge processors, we have better control over the power consumption of the system via the running average power limit (RAPL) interfaces [11, 1]. RAPL exposes on-chip energy meters for the core subsystem, processor package, and DRAM and enables the tracking of power consumption at a time resolution $(\sim 1 \mathrm{~ms})$ and system-level granularity that was not possible before. Moreover, it facilitates deterministic control over the power consumption of subsystems through power-limiting interfaces. These interfaces allow a user to specify a power bound and a time window over which the bound should be maintained. While this hardware-enforced power limiting is an appealing option, the impact of power limiting on the performance, power, and energy efficiency of enterprise-class server workloads is still not well understood and remains an active research area.

In this paper, we investigate whether it is possible to achieve energy-proportional operation for an enterprise-class server workload, namely the SPECpower_ssj2008 benchmark (hence-

\footnotetext{
${ }^{1}$ The core subsystem includes components such as the ALUs, FPUs, L1, and L2 caches 2 .

${ }^{2}$ The uncore subsystem includes components such as the memory controller, integrated I/O, and coherence engine 2 .
} 
forth referred to as SPECpower) by using the RAPL interfaces. To this end, this paper makes the following contributions:

- Insights into the mechanisms of power management for enterprise-class server workloads using the RAPL interfaces via an analysis of the SPECpower benchmark by calibrating its input parameters.

- A rigorous quantification of the energy proportionality of each subsystem within a server node via an analysis of power-consumption profiles of the different subsystems when running SPECpower at different load levels.

- The identification of system activity based on performancecounter traces that strongly correlates with subsystemlevel power consumption captured via the RAPL interfaces.

- An analysis and characterization of the instantaneouspower profiles at different load levels of SPECpower to understand the time resolution at which power limiting should be applied.

- Empirical results on the impact of RAPL power limiting on power, performance, per-transaction response time, and energy efficiency.

Through our contributions, we make the following observations and conclusions on the power management of the SPECpower benchmark using RAPL interfaces. First, the core is the most energy-proportional subsystem and the uncore is the least. Second, there is the opportunity for limiting the power consumption of the core and processor package subsystems at a resolution less than $50 \mathrm{~ms}$ for different configurations of SPECpower. Third, as the load level increases, the ability to limit the power consumption at the DRAM-level decreases. Fourth, power limiting at the level of the core subsystem is the best option for improving energy efficiency and achieving energy proportionality. Fifth, even when power limiting at the processor package-level, most of the power savings comes from the core subsystem. Sixth, better power-management mechanisms are required to achieve energy proportionality at the uncore subsystemlevel. Seventh, though we were not able to achieve energy proportionality at the full system level, i.e., entire compute node, we show that energy-proportional operation is possible at the granularity of subsystems over which we have control via RAPL power limiting (i.e., core subsystem, processor package, and DRAM).

The rest of the paper is organized as follows. In Section 2 we present the details of the SPECpower benchmark and Intel RAPL interfaces. Section 3 describes our analysis and characterization of average and instantaneous power consumption of all subsystems at different load levels in SPECpower and the observations from these experiments. Next, in Section 4, we limit the power consumption of SPECpower within different subsystems and present the impact of power limiting on power, performance, and energy efficiency. In Section 5 we describe the related work and we conclude in Section 6

\section{BACKGROUND}

In this section, we provide an overview of the SPECpower benchmark and its design as well as details into its configurable parameters. We then present the control and capabilities exposed by Intel's RAPL interfaces.

\subsection{Overview of SPECpower Benchmark}

SPECpower [4] is an industry-standard benchmark that measures both the power and performance of a server node. The benchmark mimics a server-side Java transaction processing application and is based on the SPECjbb2005 benchmark 3. It stresses the CPU, caches, and memory hierarchy and tests the implementations of the Java virtual machine (JVM), Just-in-time (JIT) compiler, garbage collection, and threads. The benchmark requires two systems: (1) the system under test or SUT and (2) the control and collection system (CCS) [5] with communication between the systems established via Ethernet 6] $]^{3}$ The SUT runs the workload and is connected to a power meter. The power meter, inturn, is connected to the CCS. The CCS collects the performance and power data passed to it by the SUT and power meter respectively.

The SPECpower benchmark is a graduated workload i.e., it runs the workload at different load-levels and reports the power and performance at each load-level [8]. The benchmark starts with a calibration phase, which determines the maximum throughput. The calibrated throughput is set as the throughput target for $100 \%$ load-level. The throughput target for the rest of the load-levels is calculated as a percentage of the throughput target for $100 \%$ load-level. For example, if the throughput target for $100 \%$ load-level is 100,000 , then the target for $70 \%$ load-level is $70,000,40 \%$ is 40,000 and so on. The throughput is measured in serverside Java operations per second (ssj_ops). The SPECpower benchmark is designed to produce consistent and repeatable performance and power measurements. It executes different type of transactions and the transactions are grouped together in batches for scheduling purposes. Each load-level is achieved by controlling delay between the arrival of batches.

The benchmark supports a set of configurable parameters 4 For example, the maximum target throughput and the batch size can be manually configured. We refer the reader to 7 . for further information on configurable parameters. The flexibility, coupled with the consistency and repeatability of SPECpower, allows us to evaluate the applicability of newer power-management interfaces, such as RAPL, to enterpriseclass server workloads.

\subsection{Intel's Running Average Power Limit (RAPL) Interfaces}

RAPL was introduced in Intel Sandy Bridge processors. The RAPL interfaces provide mechanisms to enforce power consumption limits on a specific subsystem. The only official documentation available for these interfaces is section 14.7 of the Intel software developer's manual 11. Our experiments deal only with the Sandy Bridge server platforms.

\footnotetext{
${ }^{3}$ SUT and CCS can be the same system. Communication is established via. Ethernet only if the systems are different

${ }^{4}$ Only a subset of these parameters can be changed for complaint runs.
} 
The RAPL interfaces can be programmed using the modelspecific registers (MSRs). MSRs are used for performance monitoring and controlling hardware functions. These registers can be accessed using two instructions: (1) $r d m s r$, short for "read model-specific registers" and (2) wrmsr, short for "write model-specific registers." The msr kernel module can be used for accessing MSRs from user space in Linux environments. When loaded, the msr module exposes a file interface at $/ \mathrm{dev} / \mathrm{cpu} / \mathrm{x} / \mathrm{msr}$. This file interface can be used to read from or write to any MSR on that CPU.

According to the Intel documentation, RAPL interfaces operate at the granularity of a processor socket. The server platforms provide control over three domains (i.e., subsystems): 5 (1) package (PKG), (2) power plane 0 (PP0), and (3) DRAM. We expect PKG, PP0 and DRAM to represent the processor package (or socket), the core subsystem, and memory DIMMs associated with that socket, respectively. The $M S R \_R A P L_{-} P O W E R_{-} U N I T$ register contains the units for specifying time, power, and energy, and the values are architecture-specific. For example, our testbed requires and reports time, power, and energy at increments of $976 \mathrm{mi}-$ croseconds, 0.125 watts and 15.3 microjoules, respectively. Each domain consists its own set of RAPL MSR interfaces. On a server platform, RAPL exposes four capabilities:

1. Power limiting - Interface to enforce limits on power consumption.

2. Energy metering - Interface reporting actual energy usage information.

3. Performance status - Interface reporting performance impact due to power limit.

4. Power information - Interface which provides value range for control attributes associated with power limiting.

\subsubsection{Power Limiting}

RAPL maintains an average power limit over a sliding window instead of enforcing strict limits on the instantaneous power. The advantage of having an average power limit is that if the average performance requirement is within the specified power limits the workload will not incur any performance degradation even if the performance requirement well exceeds the power limit over short bursts of time. The user has to provide a power bound and a time window in which the limit has to be maintained. Each RAPL domain exposes a MSR which is used for programming these values. The PKG domain provides two power limits and associated time window for finer control over the workload performance whereas other domains provide only one power limit. The interface provides a clamping ability, which when enabled, allows the processor to go below an OS-requested P-state.

\subsubsection{Energy Metering}

Each domain exposes a MSR interface that reports the energy consumed by that domain. On a server platform, we expect the that (1) energy $(P K G)=$ energy consumed by

\footnotetext{
${ }^{5}$ Note: We use RAPL domain and subsystem interchangeably in rest of the paper.
}

\begin{tabular}{|c|c|c|c|}
\hline Domain/Range & MTW & MaxP & MinP \\
\hline \hline Package & $45.89 \mathrm{~ms}$ & 180 watts & 51 watts \\
\hline DRAM & $39.06 \mathrm{~ms}$ & 75 watts & 15 watts \\
\hline
\end{tabular}

Table 1: Per-Socket Parameter Range (MTW = Maximum Time Window, MaxP $=$ Maximum Power, MinP = Minimum Power). Multiply by 2 For Full System

the processor package, $(2)$ energy $(\mathrm{PPO})=$ energy consumed by core subsystem, and (3) energy(uncore subsystem) $=$ en$\operatorname{ergy}(P K G)-\operatorname{energy}(P P O)$.

\subsubsection{Performance Status}

This MSR interface reports the total time for which each domain was throttled (i.e., functioning below the OS-requested P-state) due to the enforced power limit. This information will be useful in understanding the effects of power limiting on a particular workload.

\subsubsection{Power Information}

The PKG and DRAM domains expose a MSR interface that provides information on the ranges of values which can be specified for a particular RAPL domain for limiting its power consumption. This includes maximum time window, maximum power, and minimum power. The range of per-socket values on our experimental platform is given in Table 1.

\section{AN ANALYSIS OF POWER CONSUMP- TION FOR SPECPOWER}

In this section, we characterize the power consumption of the SPECpower benchmark and analyze energy proportionality from the perspective of full system as well as each RAPL domain. We then analyze performance counter traces to understand the trends seen in power consumption. Finally, we present our characterization of the instantaneous power profile of SPECpower and provide insights into the time window used for limiting the power consumption of the benchmark under different configurations. In this section, we will show the following: (1) the most and least energy-proportional subsystems are the core (PP0) and the uncore (Package$\mathrm{PP} 0$ ) respectively, (2) there is ample opportunity to limit the power consumption of SPECpower at different load-levels below the 50-ms resolution for package and PP0 domains, and (3) there is little opportunity to limit the power consumption at the DRAM-level as the load-level increases.

\subsection{Experimental Platform and Benchmark Setup}

The SUT for our experiments is a Intel Xeon E5-2665 processor (Intel Romley-EP). The node has two such processors for a total of 16 cores and 32 cores when hyperthreading is $\mathrm{ON}$, as in our experiments. It has $256 \mathrm{~GB}$ of memory and runs a Linux kernel version 3.2.0. The CCS is a Intel Xeon E5405 processor. The node has 8 cores and 8 GB of RAM. The CCS runs a Linux kernel version 2.6.32. The CCS and SUT were connected through a gigabit Ethernet network. To measure power, we used a Yokogawa WT210 power meter.

We used all the cores in SUT for our experiments. Eight warehouses with 4 threads for each warehouse was used as 
Table 2: Performance Counters Used

\begin{tabular}{|l|l|}
\hline Last-level cache references/second (LLCR) & Last-level cache misses/second (LLCM) \\
\hline Instructions retire/second (IR) & Unhalted core clock cycles (UC) \\
\hline Number of offcore outstanding reads/second (NOUT) & $\begin{array}{l}\text { Cycles in which there was one or offcore outstanding reads } \\
\text { (COUT) }\end{array}$ \\
\hline $\begin{array}{l}\text { Misses in DTLB loads that cause a page walk/second (DTL- } \\
\text { BLM) }\end{array}$ & $\begin{array}{l}\text { Misses in DTLB stores that cause a page walk/second } \\
\text { (DTLBSM) }\end{array}$ \\
\hline $\begin{array}{l}\text { Cycles spent in page walks due to DTLB load misses (DTL- } \\
\text { BLC) }\end{array}$ & $\begin{array}{l}\text { Cycles spent in page walks due to DTLB store misses } \\
\text { (DTLBSC) }\end{array}$ \\
\hline Misses in ITLB that cause a page walk/second (ITLBM) & Cycles spent in page walks due to ITLB misses (ITLBC) \\
\hline
\end{tabular}

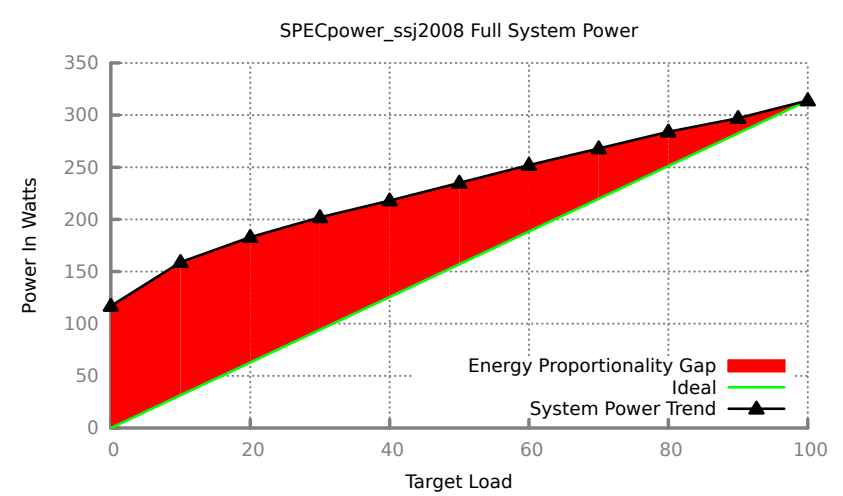

Figure 1: Illustration of Energy Proportionality Gap

the configuration for SPECpower. The 4 threads in each warehouse were pinned to two adjacent physical cores on the SUT using numactl. In order to provide consistent performance results throughout our experiments, we configured the input.load_level.target_max_throughput parameter to achieve same performance for each run. It was set to 115375 ssj_ops for each warehouse for a total of 923000 ssj_ops for the entire run. In all our experiments, $100 \%$ load-level corresponds to 923000 ssj_ops. This value was determined by averaging 10 calibration runs.

We changed the runtime for each load-level to 120 seconds using the input.load_level.length_seconds parameter and the pre- and post-measurement interval to 15 seconds in order to reduce the total runtime of the benchmark. On average, our testbed consumes 117 watts at idle and 314 watts at $100 \%$ load-level of SPECpower. We would like to stress that the system consumes $37.2 \%$ of peak power when idling.

\subsection{Energy Proportionality Metric}

As discussed earlier, we are interested in analyzing the energy proportionality of the system. The deviation of the power curve of the system from the ideal power curve is of particular interest to us. To illustrate with an example, Figure 1 shows the average power consumed by the testbed at each workload and the hypothetical/ideal energy proportional power curve for the system. The red shaded region is the deviation of interest. Henceforth, this area will be referred to as energy proportionality gap (EPG). We quantify the EPG using the EP metric. EP metric is calculated as one minus the ratio of EPG (the red region in the figure) and the area under the ideal curve 25. A value of 1 for the metric represents an ideal energy-proportional system.
A value of 0 represents a system that consumes a constant amount of power irrespective of the percentage of load-level. In our discussions, we calculate the metric by calculating the area under curves.

\subsection{An Analysis of Subsystem-Level Power Con- sumption}

In this section, we present the details on the power consumption of SPECpower at a subsystem-level. We were able to profile the benchmark at a granularity which has not been possible until the advent of Intel Sandy Bridge by using the on-chip energy meters exposed by the RAPL interfaces. Our results provide insights into the energy proportionality of a system as a whole as well as at the RAPL domain-level. We also present the details of the power consumed using different batching sizes. Batching queries to exploit and create opportunities for power management is a well-researched area 20]. The number of transactions in each batch scheduled of the SPECpower benchmark is calibrated using the input.scheduler.batch_size input parameter. We use four different batching sizes (i.e, 1000, 5000, 7500 and 10000) in each of our experiments.

\subsubsection{Methodology}

We used the energy meters exposed in each RAPL domain to determine the power dissipated at each domain. We also collected specific performance counter data which correspond to RAPL domain and full system power in order to give more insights into the power profile of SPECpower. Table 2 shows the list of performance counters we profiled. We determined these performance counters based on previous work 22,14 and we use newer ones that might have effects on power curve of the system.

Since we have access to energy meters at different RAPL domains, we are also interested in finding the performance counters that affect the power curve of that domain. Specifically, we want to find the performance counters which have correlation with the power consumption of each domain. This study will help guide the modeling the power consumption of different load-levels at a subsystem-level. Principal Component Analysis (PCA) is used to determine the most significant performance counter for particular RAPL domain as well as full system. Note that the correlation analysis is performed before performing PCA in order to obtain performance counters that show good correlation to power. PCA is widely used for dimension reduction in many areas. The main concept behind PCA is to reduce the number of dimensions in a data set while preserving the variance of the data set as much as possible. This is achieved by transforming the 

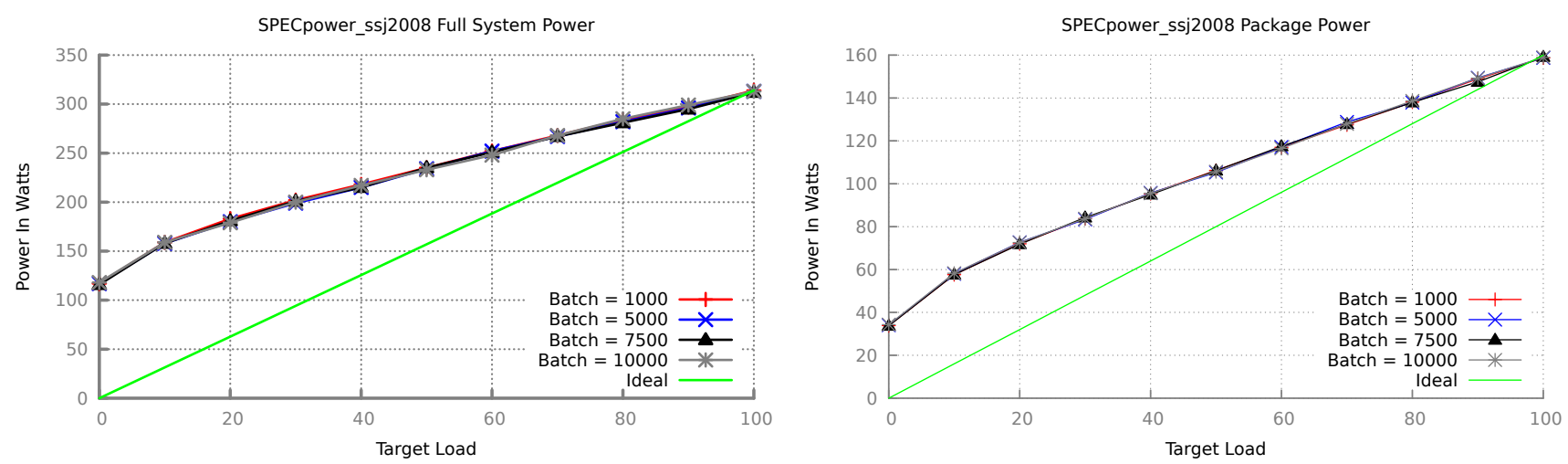

Figure 2: Power Profiles - Left:Full System, Right:Package
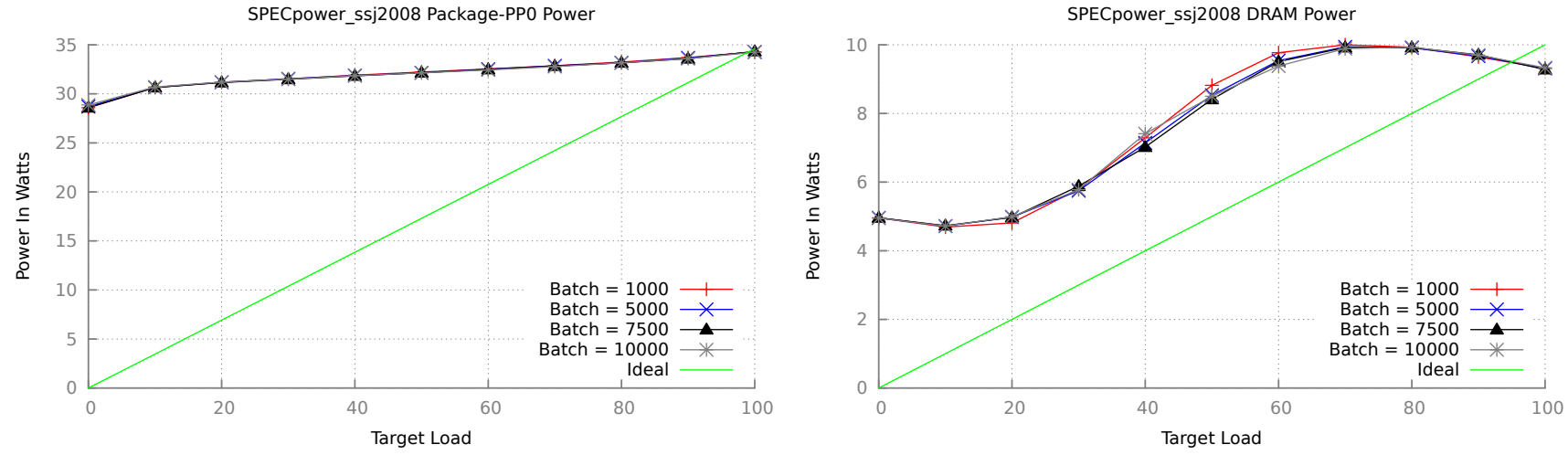

Figure 3: Power Profiles - Left:Uncore (Package-PP0), Right:DRAM

data into principal components (PCs). The principal components are linear combinations of the original set of variables and are uncorrelated to each other. We first normalize the performance counter data to a unit normal distribution to mitigate the effect of varying range of values for different performance counters and then apply PCA. We use R to perform these statistical analyses in our experiments.

\subsubsection{Profiling of the Full System and Package Power}

Figure 2 shows the power profile for the full system and package RAPL domain while running SPECpower using different batch sizes. In both cases, the batch size does not affect the average power profile of the system. It is also worth noting that both the power profile follow a similar trend indicating a strong correlation between full system and package power (Pearson correlation is greater than 0.99). Consequently, the power consumed by the domains have a strong correlation $(>0.95)$ with the same performance counters. Both these power profiles have a strong correlation with all counters except the counters related to ITLB in Table 2 Our PCA reveals that five PCs (LLCM, IR, NOUT, DTLBLM and DTLBSM) account for $95 \%$ of the variance.

The EP metric value for the full system is 0.54 for all batch sizes whereas the metric value for package domain is 0.71 for all batch sizes. Comparison of the EP metric shows that EPG for package domain is smaller than the full system suggesting that package domain is more energy-proportional than the full system.

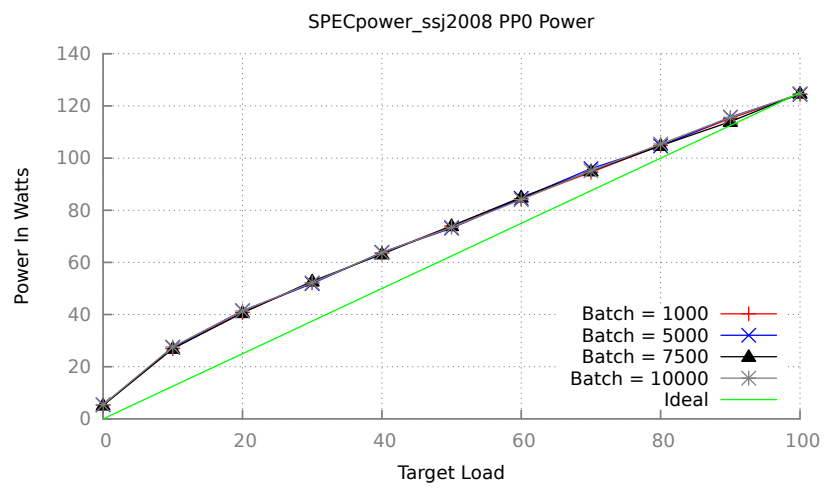

Figure 4: Power Plane 0 (PP0) Power Profile

\subsubsection{Profiling of the Uncore (Package-PPO) and DRAM Power}

The uncore subsystem and DRAM power profiles are shown in Figure 3 . The counters except the ones related to ITLB and DTLB stores have a correlation greater than 0.80 with power consumption of the uncore subsystem. Our PCA revealed that four PCs (IR, NOUT, DTLBLM and LLCM) 
account for $83 \%$ of the variance. Our analysis reveals that the counters in Table 2 are not enough to model the power consumption of uncore subsystem. We intend to look at uncore performance counters 2] exposed in the Sandy Bridge server platforms to uncover performance counters with better correlation in the future. The uncore subsystem power remains almost a constant irrespective of the $\%$ of the loadlevel with an EP metric value of 0.17 . The uncore subsystem has the greatest EPG and as a result exhibits the worst power consumption trend among the full system and RAPL domains from the perspective of energy-proportional power scaling.

Interestingly, the DRAM domain consumed a maximum average power of only 10 watts (i.e., 5 watts per socket). The performance counters related to LLC, DTLB, instructions retired and outstanding bus requests have a correlation greater than 0.91 with the power consumed by DRAM. Four PCs (LLCM, IR, DTLBLM and NOUT) retain $91 \%$ of the variance. The DRAM domain's value for EP metric is 0.47 . The SPECpower benchmark only consumes a maximum of approximately 10 watts for the DRAM domain. Moreover, the benchmark consistently consumes it's maximum power for the DRAM domain at $70 \%$ workload for all batches.

\subsubsection{Profiling of the Power Plane O (PPO) Power}

Figure 4 represents the power profile of PP0 domain. Similar to other domains, the batch size does not affect the average power consumption of the domain. All counters except the ones related to ITLB and LLC have strong correlation with the power profile of this domain. Four PCs (IR, NOUT, DTLBLM and DTLBSM) account for $97 \%$ of all variance. $\mathrm{PP0}$ is the most energy proportional subsystem with an EP metric value of 0.86 across all batches. Interestingly, the idle power consumption of PP0 domain is 5.27 watts. The PP0 domain has the least EPG and exhibits near energyproportional power scaling.

\subsection{An Analysis of Subsystem-Level Instanta- neous Power Consumption}

In this section, we present our results for instantaneous power profile analysis of SPECpower benchmark. Our main goal is to visualize the opportunities for power limiting under different time window while consuming power proportional to the load-level. We collected instantaneous power profile for three load-levels (50, 60 and 70) and four different batch sizes of SPECpower using a loadable kernel module. The resolution of our power profile is limited to 5 milliseconds (ms). Our instrumentation had negligible impact on the power and performance of the SPECpower benchmark.

\subsubsection{Methodology}

Figures 5, 6 \& 7] present the results on the instantaneous power profile analysis of SPECpower. These plots describe the opportunity for power limiting under different time windows while consuming power proportional to the load-level. The $\mathrm{x}$-axes in the graphs is the resolution of time for which we profile the instantaneous power profile. The y-axes represents the ratio of data points for which the instantaneous power profile was greater than ideal energy proportional power consumption and the total number of data points for that time resolution. For example, consuming $50 \%$ and $70 \%$ of power at $100 \%$ load-level is considered energy-proportional operation for $50 \%$ and $70 \%$ load-levels respectively. Henceforth, we refer to the ideal power consumption for a loadlevel as LL\% of peak power. As an example, in Figure 5 at $50 \mathrm{~ms}$ resolution using 1000 as batch size, the instantaneous power was over ideal energy-proportional power consumption for 99.58, 97.25 and 93.25 percent for 50, 60 and $70 \%$ load-levels respectively. Performance is a implicit metric when considering energy-proportional operation. Consequently, we are interested in time resolutions which will allow us to achieve energy-proportional operation with minimal or no impact on performance. These graphs can be used to determine the time window(s) useful to limit the power consumption for a certain batch size and load-level to achieve energy proportional operation. Note that we restrict our analysis to 50, 60 and $70 \%$ load-level for simplicity. However, our methodology is applicable to all batch sizes and load-levels.

\subsubsection{Results}

Package: Figure 5 presents the results for the instantaneous power profile analysis of package RAPL domain. More than $95 \%$ of the data points consume greater than $\mathrm{LL} \%$ of peak power for all load-levels with batch size $=1000$ at a resolution greater than $100 \mathrm{~ms}$. This limits the opportunity to limit the power to achieve energy proportional operation at time windows greater than $100 \mathrm{~ms}$. However, increase in batch size of SPECpower improves the opportunity to do power limiting at bigger time resolution. We also observe that this improvement is higher for bigger load-levels. For example, change in data points which consumes LL\% of peak power moves from $99.83 \%, 99.66 \%$ and $96.16 \%$ to $97.16 \%, 92.00 \%$ and $81 \%$ for 50,60 and 70 percent workloads, respectively, for change in batch sizes from 1000 to 10000 .

PP0: Figure 6 presents the results for the instantaneous power profile analysis of the PP0 RAPL domain. In general, PP0 provides better opportunities for power limiting at different time resolutions when compared to the package domain. This is expected as the uncore subsystem consumed almost constant power irrespective of the load-level as discussed in Section 3.3.3 Even using batch size as 1000, there are significant opportunities to limit power to LL\% of peak power at granularities less $250 \mathrm{~ms}$ for 60 and 70 percent load-levels. Similar to package domain increase in batch size improves the opportunity to limit power at bigger resolution. However, the improvement is higher when compared to the package domain. For example, change in data points which consumes LL\% of peak power moves from $92.83 \%, 80.33 \%$ and $72.33 \%$ to $79.00 \%, 70.33 \%$ and $52.66 \%$ for 50,60 and 70 percent workloads, respectively, for change in batch sizes from 1000 to 10000 .

DRAM: Figure 7 shows the results for the instantaneous power profile analysis of DRAM RAPL domain. In contrast to the package and PP0 RAPL domains, the opportunity for power limiting at higher time resolution is more at lower load-levels. We observe that for $60 \%$ and $70 \%$ load-levels approximately $90 \%$ of the data points consume more than LL\% of peak power for all different batch sizes. This shows that the opportunity to achieve energy-proportional DRAM operation for these load-levels through power limiting is lim- 
Package Instantaneous Power

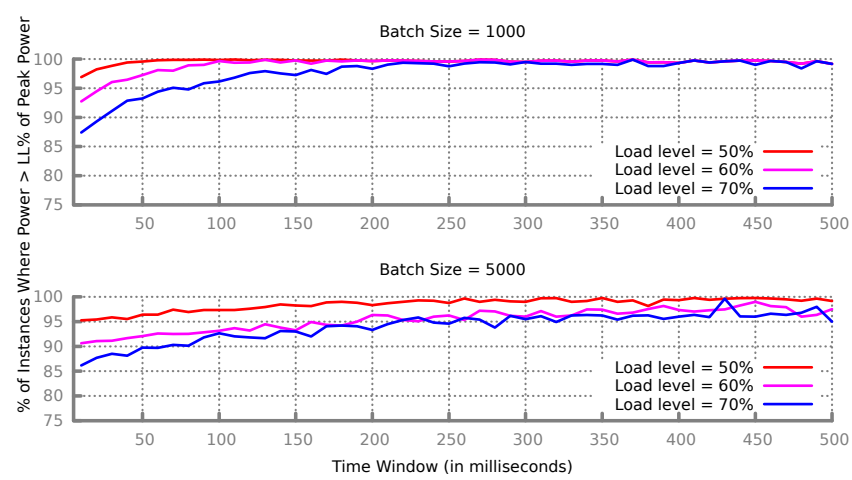

Package Instantaneous Power

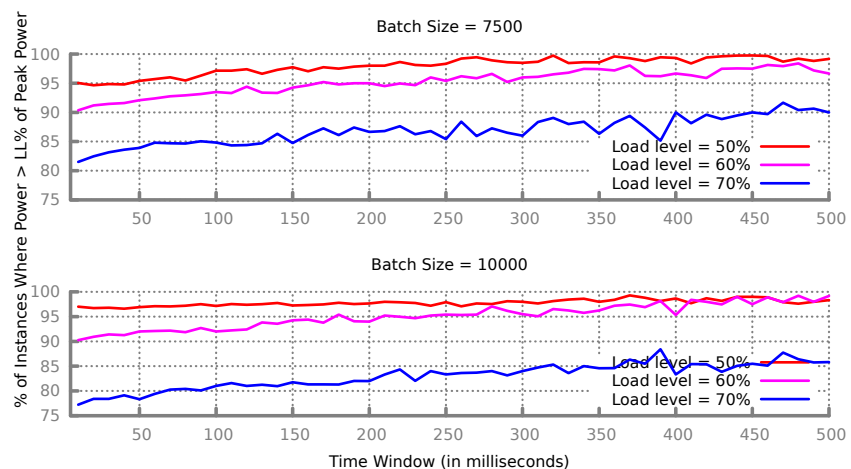

Figure 5: Instantaneous Power Analysis For Package Domain

PPO Instantaneous Power

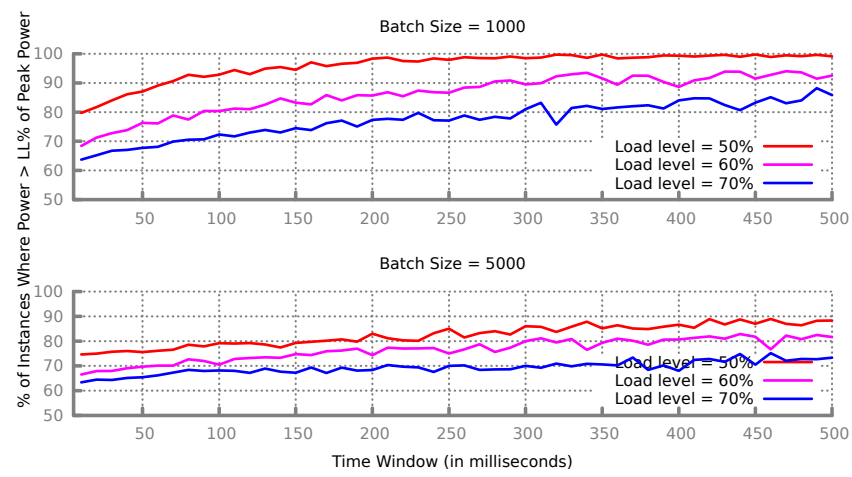

PPO Instantaneous Power

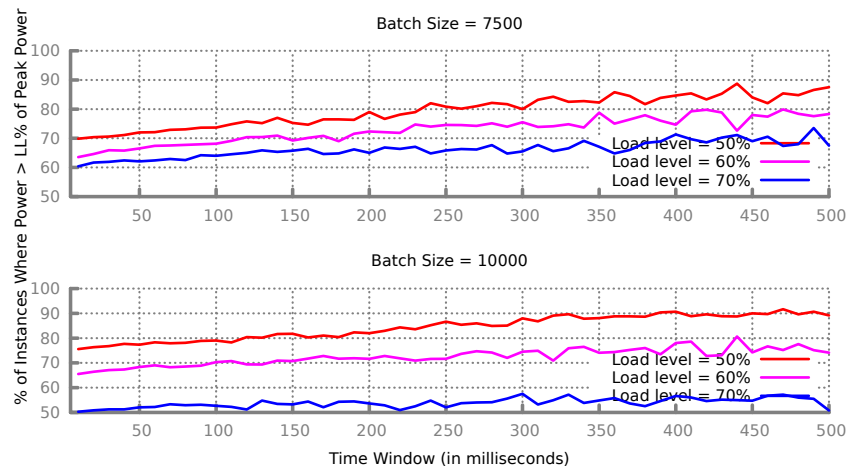

Figure 6: Instantaneous Power Analysis For PP0 Domain
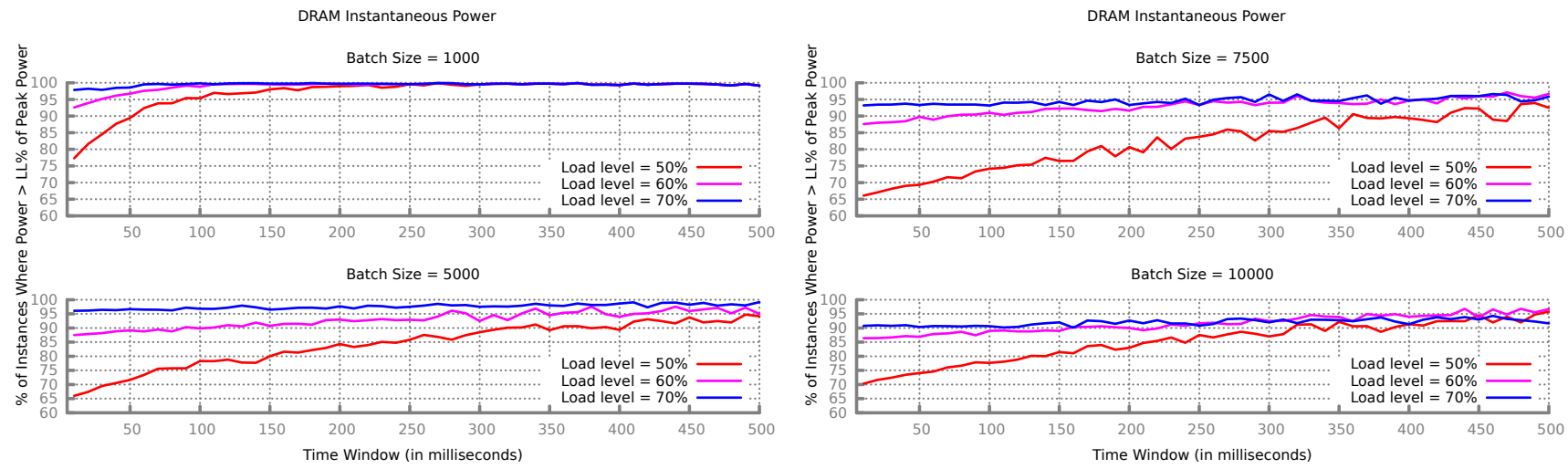

Figure 7: Instantaneous Power Analysis For DRAM Domain

ited. However, $50 \%$ load-level is amenable to power limiting in this RAPL domain. Similar to Package and PP0, increase in batch size allows power limiting even at bigger resolution. However, the improvement is better for lower load-levels i.e., $50 \%$ load has more opportunity for power limiting than $60 \%$ or $70 \%$ load-levels at bigger time resolutions.

\section{IMPACT OF POWER LIMITING ON SPECPOWER}

In this section, we discuss the effects of power limiting on the performance and power of SPECpower. Specifically, we leverage these RAPL interfaces and analyze whether energyproportional operation can be achieved. While we are not able to achieve energy proportionality at full system-level, RAPL power limiting is useful to improve energy efficiency without performance degradation. In addition, we are able to achieve energy-proportional operation or better at packageand memory-level (the subsystems over which we have power limiting control) for the load-levels discussed. 

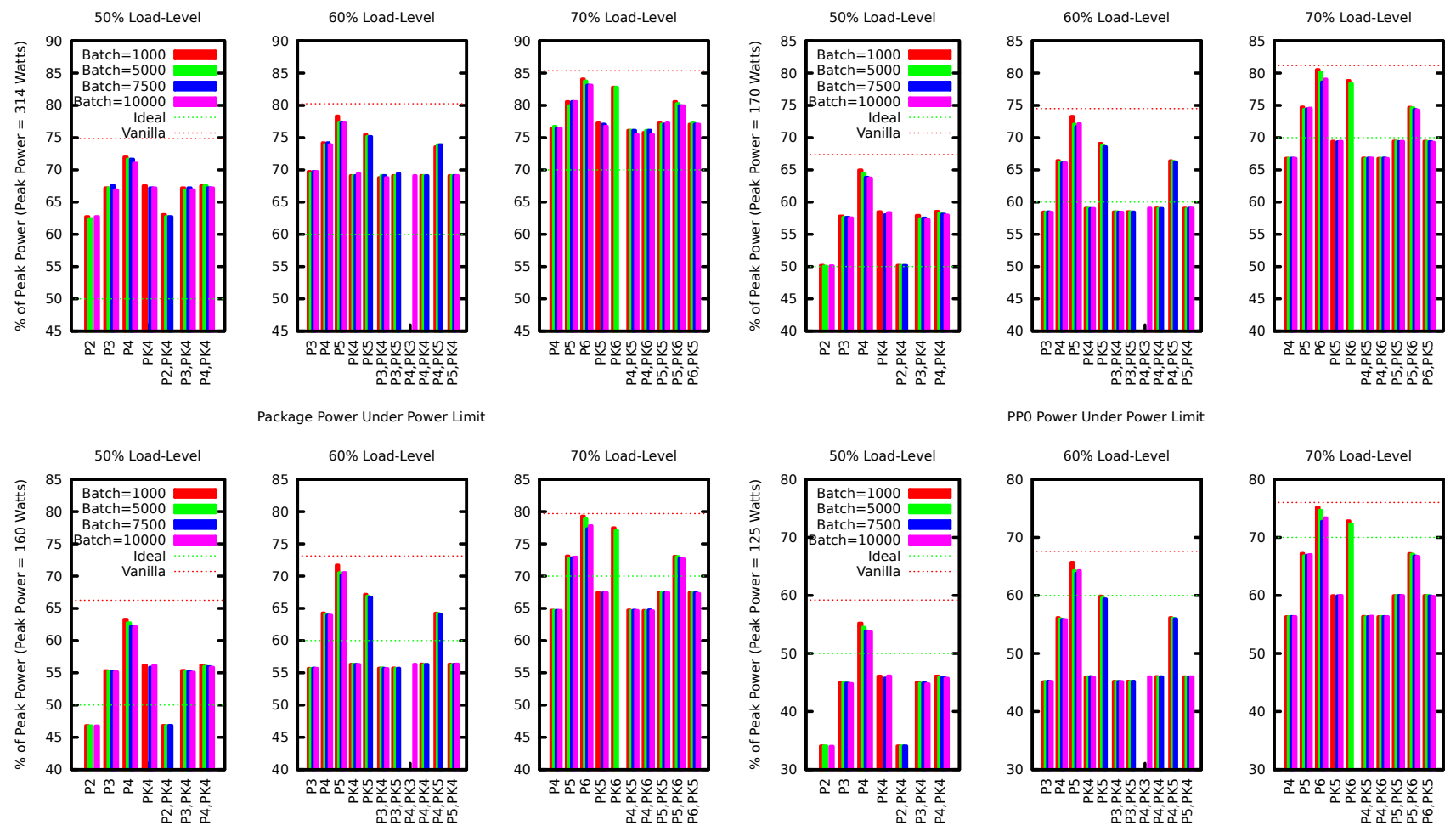

Figure 8: Power Consumption Under Power Limit

\subsection{Methodology}

We run 50, 60 and 70 percent load-levels of SPECpower under power limits by manually configuring the power limiting interfaces. We were not able to use the power limiting control for the DRAM domain for SPECpower because the maximum average DRAM power consumption (see Section 3.3.3) is less than the allowed minimum power consumption for the DRAM domain (see Section 2.2.4). Experiments specifying relevant power consumption limit for the DRAM domain for SPECpower resulted in no effect on power. As a results, our experiments will focus only on limiting the power consumption of Package and PP0 RAPL domains. We manually limit the power consumption of these domains to different values of power for different load-levels as shown in Table 3

\begin{tabular}{|l|l|l|}
\hline $\begin{array}{l}\text { Load- } \\
\text { Level/Domain }\end{array}$ & PP0 & Package \\
\hline 50 & $\begin{array}{l}20,30,40,50(\mathrm{P} 1, \\
\text { P2, P3, P4) }\end{array}$ & $\begin{array}{l}25,30,40,50(\mathrm{PK} 1, \\
\text { PK2, PK3, PK4) }\end{array}$ \\
\hline 60 & $\begin{array}{l}30,40,50,60(\mathrm{P} 2, \\
\mathrm{P} 3, \mathrm{P} 4, \mathrm{P} 5)\end{array}$ & $\begin{array}{l}30,40,50,60(\mathrm{PK} 2, \\
\mathrm{PK} 3, \mathrm{PK} 4, \mathrm{PK} 5)\end{array}$ \\
\hline 70 & $\begin{array}{l}40,50,60,70(\mathrm{P} 3, \\
\mathrm{P} 4, \mathrm{P} 5, \mathrm{P} 6)\end{array}$ & $\begin{array}{l}40,50,60,70(\mathrm{PK} 3, \\
\mathrm{PK} 4, \mathrm{PK} 5, \mathrm{PK} 6)\end{array}$ \\
\hline
\end{tabular}

Table 3: Power Limit Configuration For Each loadlevel (In \% of Peak Power For That Domain)

\subsection{Impact on Power Consumption}

Figure 8 portrays the power consumption of SPECpower under power limits for three different load-levels. We present power consumption results for the full system and three subsystems 1. full system, 2. Package+DRAM (the domains over which we have power limiting control), 3. Package and 4. PP0. Each plot presents only the configurations from Table 3 which achieved the particular load-level. For the purpose of comparison, we also show the average power consumption of the subsystem without power bounds (vanilla - red dotted line) and energy-proportional power (ideal green dotted line). All the results presented in Figure 8 use the least possible value as the time window for power limiting (i.e., 976 microseconds).

We observe that power limiting does not allow us to enable energy-proportional operation at the full system level. We would like to emphasize that the system consumed $37.2 \%$ of peak power (i.e., 314 watts) even when idling. However, we were able to achieve moderate power savings at the full system level without any performance degradation by using power limiting. We also observe that the power saving increases with decreased load-level. For example, the best full system power saving achieved at 50\% and $70 \%$ loadlevel are $13 \%$ and $9 \%$ of peak power over the vanilla runs of SPECpower.

We were able to achieve energy-proportional operation at the PKG+DRAM, PKG and PP0 RAPL domain levels for all load-levels. Power limiting in PP0 RAPL domain is the best possible mechanism to achieve energy-proportional operation. Interestingly, the combination of a PP0 power limit and package power limit always consumed approximately the same power as a corresponding run with only PP0 power 
limit. Our power limiting experiments with only the package domain revealed that, approximately $98.5 \%$ of the power saving came from the PP0 domain in all load-levels. The reason for such power profile was due to the fact that the uncore consumes almost constant power irrespective of the loadlevel (see Section 3.3.3). We require better mechanisms to control the power consumption of the uncore subsystem 2].

\subsection{Impact on Response Time}

The response time in workloads such as SPECpower can greatly impact user experience. In this section, we measure the impact of power limiting on per-transaction response time. The response time can vary widely even if the throughput (i.e., load-level) is maintained. The arrival time, wait time and total response time of batches in a run can be logged by setting the input.scheduler.log_arrival_rates to true. We are interested in the total response time (wait + execution time) as it is directly related to the performance as seen by a user. Figure 9 shows the average pertransaction response time slowdown of three different loadlevels and four different batch sizes under power limit. The slowdown is calculated as the ratio of response time under power limit and the response time of a vanilla run. We first observe that the response time varies widely within a given load-level as expected. PP0 power limiting gives the best power-performance trade-off. The response time is directly correlated to batch size in all load-levels. With bigger batch sizes, the response time for SPECpower decreases. The response time with batch size $=1000$ worsens as we use package power limiting.

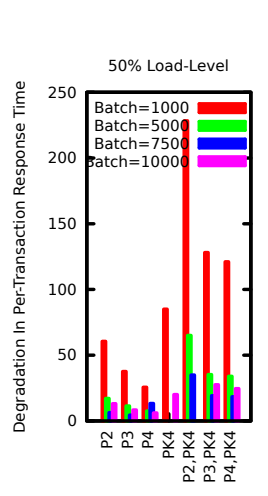

Response Time Under Power Limit
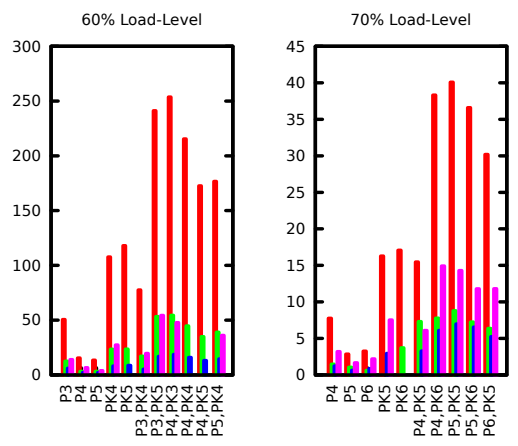

Figure 9: Response Time Under Power Limit

\subsection{Impact on Energy Efficiency}

As discussed earlier, SPECpower is a power and performance benchmark with ssj_ops/watt (i.e., performance-topower ratio) as the main metric of interest. Figure 10 depicts the energy efficiency improvement under power limits. The improvement is calculated as the ratio of difference between the energy efficiency under power limit and vanilla run over the efficiency in a vanilla run. We observe that the $\%$ gain in energy efficiency decreases with increase in load-level. Power limiting enables us to achieve a maximum energy efficiency improvement of 20,16 and 12 percent at 50, 60 and 70 percent load-levels respectively. These results show that PP0 power limiting (in isolation) is the best mechanism to achieve better energy efficiency.

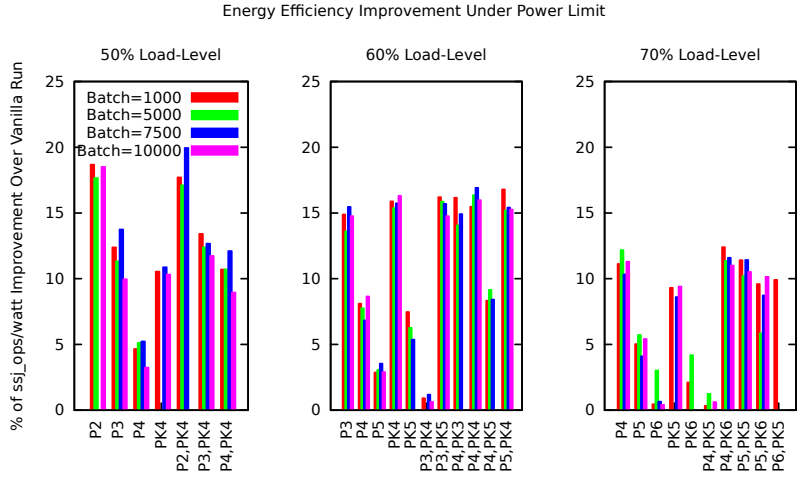

Figure 10: Energy Efficiency Improvement Under Power Limit

\subsection{Impact of Power Limiting Time Window on Response Time}

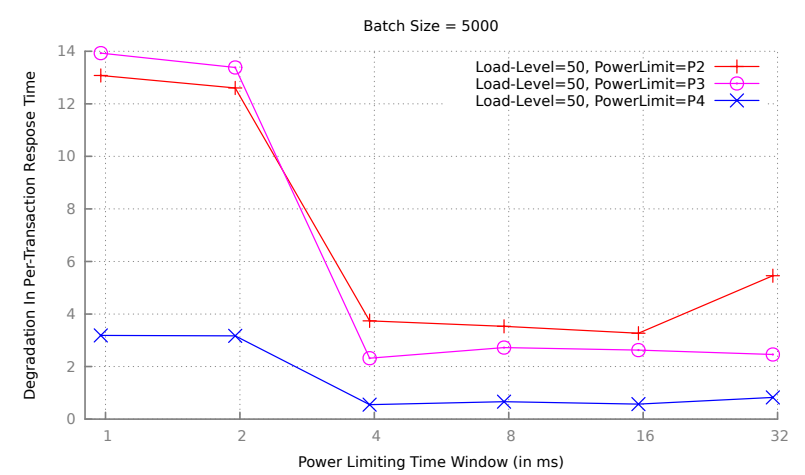

Figure 11: Impact of Power Limiting Time Window on Response Time

Figure 11 shows the impact of power limiting time window on the per-transaction response time for running SPECpower with 5000 as the batch size and setting power limit for PP0 RAPL domain. We show only one batch size and load-level for simplicity. The rest of the batch sizes and load-levels showed similar trends. We would like to emphasize that the average power consumption of SPECpower remains approximately the same for a specified power limit with different power limiting time window. There is a huge improvement moving from $\sim 2 \mathrm{~ms}$ to $\sim 4 \mathrm{~ms}$ time window. In general, increasing the time window improves the response time of SPECpower. However, the improvement stagnates after certain increase in the time window. We observe there is not much improvement in the response for all load-levels after the $4 \mathrm{~ms}$ time window.

\section{RELATED WORK}

In this section we present some of the existing literature related to our work. The related work can be divide in three categories. 1. energy-proportional operation for enterprise class workloads, 2. characterization and power analysis of SPECpower benchmark and 3. power limiting.

\subsection{Energy-Proportional Operation For Enter- prise Class Workloads}


Our work is most related to the work of Meisner et al. 21. They characterize online data intensive services (OLDI) to identify opportunities for power management, design a framework which predicts the performance of OLDI workloads and investigate the power and performance trade-offs using the framework. Our work leverages Intel's RAPL interfaces to characterize the power consumption of SPECpower and analyze whether it is possible to achieve energy-proportional operation on a system. Fan et al. 12 investigate the benefits of energy-proportional systems in improving the efficiency of power provisioning using their power models. They provide evidence which support that energy-proportional systems will enable improve power capping at the data center level. In contrast, we look at leveraging power capping mechanism to achieve energy-proportional operation for SPECpower. Tolia et al. 27] proposed that by migrating workloads from under-utilized to other systems and turning the under-utilized systems off, energy proportionality can be approximated at an ensemble-level (i.e., for a group of nodes or rack-level). They used VM migration as a software mechanism to move workloads off of under-utilized systems. In this paper, we use user-defined and hardware-enforced power limiting to achieve energy-proportional systems at node-level which is applicable to several class of server workloads.

\subsection{Charecterization and Power Analysis of SPECpower}

Fanara et al. 13 and Lange 19 comment on the design and development of SPECpower benchmark. An overview of the workload, its behavior and detailed characterization of SPECpower benchmark under different load-levels is described in [16]. Our goal in this paper is to characterize SPECpower to provide insights into the opportunities for power management for enterprise class server workload.

Hsu et al. 18 defined accurate and portable power models which captures the linearity of the power curve under different load-levels of SPECpower. Varsamopoulos et al. 28, proposed idle-to-power ratio (IDR) and linear deviation ratio (LDR) metrics, analyzed a large number of SPECpower results and provided insights into server provisioning. Similarly, Ryckbosch et al. 25. proposed the EP metric to quantify the energy proportionality of the system. In this paper, for the first time, we quantify the energy proportionality at a subsystem-level using the on-chip energy meters exposed through RAPL. We also characterize the instantaneous power profile at different load levels of SPECpower.

\subsection{Power Limiting}

Several mechanisms to cap the power consumption of the system have been studied 10, 15. However, we study the use of RAPL power limiting which is hardware-enforced in this paper. David et al. 11] proposed RAPL and evaluated RAPL for memory sub-system. They present a model which accurately predicts the power consumed by the DIMMs and use RAPL to cap the power consumption. Rountree et al. 23 use RAPL power limiting to study the behavior of performance for benchmarks in NAS parallel benchmark suite. Specifically, they are interested in the performance of various compute nodes under a power bound. Weaver et al. 29. have have exposed RAPL energy meters through
PAPI. We use RAPL interfaces to achieve energy-proportional operation for SPECpower benchmark and to the best of our knowledge, there is no previous study on using RAPL interfaces for enterprise class server workloads.

\section{CONCLUSION AND FUTURE WORK}

Power and energy management is a key issue for data centers. Efficient power management of enterprise class server workloads have the potential to greatly reduce energy-related costs and facilitate efficient power provisioning. Energy proportionality has the potential of having huge impact in improving the energy efficiency of data centers. In this paper, we investigate if it is possible to achieve energy proportionality for SPECpower benchmarks using RAPL interfaces. Our study throws light on the mechanisms for power management of enterprise class server workloads and the efficacy of RAPL interfaces. We identify the least and most energyproportional subsystem using the on-chip energy meters. Performance counter traces are collected to identify which events have strong correlation with power consumption at a subsystem-level. We characterize the instantaneous power profile of SPECpower and identify the time resolutions at which there is opportunity to limit the power consumption of the benchmark. We use RAPL power limiting and show that we are able to achieve energy-proportional operation or better at subsystem-levels.

We are interested in extending our work to create a runtime system to achieve energy-proportional operation using power limiting. Particularly, we want to address the following problems. 1. understand the power impact of increasing the memory footprint of SPECpower benchmark and how memory power limiting can help in achieving energy-proportional operation. 2. model the performance of SPECpower under subsystem-level power bounds using utilization as a metric which includes defining the utilization of a subsystem (e.g., DRAM or core subsystem), 3. formulate a multi-dimensional optimization problem to find the best possible power limit and time window for each subsystem using the performance model under a performance degradation limit and 4. create a runtime system using the power models and the optimization framework.

\section{REFERENCES}

[1] Intel 64 and IA-32 Software Developer Manuals Volume 3. Available at www.intel.com/content/www/us/en/processors/ architectures-software-developer-manuals.html.

[2] Intel Xeon Processor E5-2600 Product Family Uncore Performance Monitoring Guide. Available at http://www.intel.com/content/dam/www/public/ us/en/documents/design-guides/ xeon-e5-2600-uncore-guide.pdf

[3] SPECjbb Benchmark. Available at http://www.spec.org/jbb2005/

[4] SPECpower Benchmark. Available at http://www.spec.org/power_ssj2008

[5] SPECpower Benchmark - CCS Design Document. Available at http://www.spec.org/power/docs/ SPECpower_ssj2008-Design_ccs.pdf.

[6] SPECpower Benchmark - Design Overview. Available at http://www . spec .org/power/docs/SPECpower _ 
ssj2008-Design_Overview.pdf

[7] SPECpower Benchmark - Run Rules. Available at http://www . spec.org/power/docs/SPECpower ssj2008-Run_Reporting_Rules.html

[8] SPECpower Benchmark - SSJ Workload Design Document. Available at http://www.spec.org/power/ docs/SPECpower_ssj2008-Design_ssj.pdf

[9] L. A. Barroso and U. Hölzle. The Case for Energy-Proportional Computing. IEEE Computer, 40(12):33-37, 2007.

[10] R. Cochran, C. Hankendi, A. K. Coskun, and S. Reda. Pack \& Cap: Adaptive DVFS and Thread Packing Under Power Caps. In Proceedings of the International Symposium on Microarchitecture, MICRO-44, pages 175-185, 2011.

[11] H. David, E. Gorbatov, U. R. Hanebutte, R. Khanna, and C. Le. RAPL: Memory Power Estimation And Capping. In Proceedings of the International Symposium on Low Power Electronics and Design, ISLPED, pages 189-194, 2010.

[12] X. Fan, W.-D. Weber, and L. A. Barroso. Power Provisioning For a Warehouse-Sized Computer. In Proceedings of the International Symposium on Computer Architecture, ISCA, 2007.

[13] A. Fanara, E. Haines, and A. Howard. The State of Energy and Performance Benchmarking for Enterprise Servers. In R. Nambiar and M. Poess, editors, In Performance Evaluation and Benchmarking, pages 52-66. Springer-Verlag, 2009.

[14] M. Ferdman, A. Adileh, O. Kocberber, S. Volos, M. Alisafaee, D. Jevdjic, C. Kaynak, A. D. Popescu, A. Ailamaki, and B. Falsafi. Clearing the Clouds: A Study of Emerging Scale-out Workloads on Modern Hardware. In Proceedings of International Conference on Architectural Support for Programming Languages and Operating Systems, ASPLOS, pages 37-48, 2012.

[15] A. Gandhi, M. Harchol-Balter, R. Das, J. Kephart, and C. Lefurgy. Power Capping Via Forced Idleness. In Proceedings of Workshop on Energy-Efficient Design, WEED, 2009.

[16] L. Gray, A. Kumar, and H. Li. Workload Characterization of the SPECpower_ssj2008 Benchmark. In Performance Evaluation: Metrics, Models and Benchmarks, Lecture Notes in Computer Science, pages 262-282. 2008.

[17] C. Hsu and W. Feng. A Power-Aware Run-Time System for High-Performance Computing. In In Proceedings of the SC Conference, 2005.

[18] C. Hsu and S. W. Poole. Power signature Analysis of the SPECpower_ssj2008 Benchmark. In Proceedings of the International Symposium on Performance Analysis of Systems and Software, ISPASS, pages 227-236,
2011.

[19] K. Lange. Identifying Shades of Green: The SPECpower Benchmarks. Computer, 42(3):95-97, Mar. 2009.

[20] D. Meisner, B. T. Gold, and T. F. Wenisch. PowerNap: Eliminating Server Idle Power. In Proceedings of International Conference on Architectural Support for Programming Languages and Operating Systems, ASPLOS, 2009.

[21] D. Meisner, C. M. Sadler, L. A. Barroso, W.-D. Weber, and T. F. Wenisch. Power Management of Online Data-Intensive Services. In Proceedings of the International Symposium on Computer Architecture, ISCA, pages 319-330, 2011.

[22] S. Rivoire. Models and Metrics for Energy-Efficient Computer Systems. Ph.D. Dissertation, Department of Electrical Engineering, Stanford, 2008.

[23] B. Rountree, D. Ahn, B. de Supinski, D. Lowenthal, and M. Schulz. Beyond DVFS: A First Look at Performance Under a Hardware-Enforced Power Bound. In Proceedings of International Parallel and Distributed Processing Symposium Workshops and PhD Forum, IPDPSW, pages 947-953, 2012.

[24] B. Rountree, D. K. Lownenthal, B. R. de Supinski, M. Schulz, V. W. Freeh, and T. Bletsch. Adagio: Making DVS Practical For Complex HPC Applications. In Proceedings of the International Conference on Supercomputing, ICS, pages 460-469, 2009.

[25] F. Ryckbosch, S. Polfliet, and L. Eeckhout. Trends in Server Energy Proportionality. IEEE Computer, (9):69-72, 2011.

[26] D. C. Snowdon, S. M. Petters, and G. Heiser. Accurate On-line Prediction of Processor and Memory Energy Usage Under Voltage Scaling. In Proceedings of the International Conference on Embedded Software, EMSOFT, pages 84-93, 2007.

[27] N. Tolia, Z. Wang, M. Marwah, C. Bash, P. Ranganathan, and X. Zhu. Delivering Energy Proportionality with Non Energy-Proportional Systems: Optimizing the Ensemble. In Proceedings of the Conference On Power Aware Computing and Systems, HotPower. USENIX Association, 2008.

[28] G. Varsamopoulos, Z. Abbasi, and S. Gupta. Trends and Effects of Energy Proportionality on Server Provisioning in Data Centers. In Proceedings of the International Conference on High Performance Computing, HiPC, pages 1-11, 2010.

[29] V. Weaver, M. Johnson, K. Kasichayanula, J. Ralph, P. Luszczek, D. Terpstra, and S. Moore. Measuring Energy and Power with PAPI. In International Workshop on Power-Aware Systems and Architectures, PASA, 2012. 